\title{
Le temps atomique
}

\author{
Thierry BASTIN
}

Département de Physique, Université de Liège

\section{Introduction}

Le temps est cette notion qui vise à rendre compte de l'évolution des choses. Dans le monde qui nous entoure, rien ne semble statique, tout évolue, change. Pour quantifier ces évolutions, les comparer entre elles et établir en particulier des lois physiques, l'homme s'ingénie à ce qu'il appelle «mesurer le temps qui passe », à déterminer (de manière quantitative) la «durée des phénomènes physiques ». Que cela signifie-t-il exactement? De manière générale, la mesure d'une grandeur physique consiste simplement à comparer cette grandeur avec une grandeur de même nature, prise comme référence et servant de ce fait d'unité de mesure pour la grandeur physique en question. On compare des pommes avec des pommes et des poires avec des poires... Rien de plus. Par exemple, la mesure d'une dimension (longueur) consiste à comparer la dimension en question avec une dimension de référence, c'est-à-dire à déterminer par quelque procédé que ce soit quel est la multiple $x$ de la dimension de référence qui reproduit la dimension à déterminer. La dimension de référence, qui porte le nom d'unité de longueur, est évidemment totalement arbitraire. Pour d'évidentes raisons de communication des résultats de mesure, il est toutefois important que tout le monde utilise la même dimension de référence, c'est-à-dire la même unité de longueur. C'est actuellement le cas et il a été donné le nom de mètre à cette unité de longueur adoptée internationalement par tous et dont la définition a varié à plusieurs reprises. La dimension intrinsèque de cette unité de longueur ne revêt aucune propriété particulière et l'histoire aurait pu produire une unité de longueur portant toujours le nom de mètre, mais différente de celle universellement utilisée aujourd'hui.

Pour la mesure du temps, le principe est rigoureusement le même. Mesurer la durée d'un phénomène ne consiste en rien d'autre qu'à comparer la durée du phénomène en question par rapport à la durée d'un autre phénomène, prise comme durée de référence et portant le nom d'unité de temps. Tout comme l'unité de longueur, l'unité de temps est a priori totalement arbitraire. Sa définition a par ailleurs varié au cours de l'histoire. Jusqu'en 1960, l'unité de temps, dénommée la seconde, a été tacitement définie comme étant la durée d' $1 / 86400^{\mathrm{e}}$ de jour solaire moyen, soit, en simplifiant un peu, $1 / 86400^{\mathrm{e}}$ du temps 
moyen $^{l}$ requis par le Soleil pour effectuer 2 passages successifs au méridien du lieu d'observation, ou encore, dit autrement, $1 / 86400^{\mathrm{e}}$ de la durée moyenne requise à la Terre pour accomplir une rotation autour d'elle-même jusqu'à revoir le Soleil exactement plein sud comme la veille. Compte tenu du mouvement de la Terre autour du Soleil, le jour solaire moyen n'est rien d'autre qu'un multiple bien déterminé (un peu au-dessus de 1) de la durée d'une rotation complète (de $360^{\circ}$ ) de la Terre sur elle-même (le jour sidéral). La seconde d'avant 1960 correspond donc tout simplement à une fraction donnée du jour sidéral. Il s'agit d'une durée de référence en lien direct avec la rotation de la Terre sur ellemême. Cette définition n'a de sens que si le jour sidéral reste constant. Or il n'en est rien et des études poussées dans les années 1950 ont mis en évidence de nombreuses irrégularités dans la période de rotation de la Terre sur elle-même. Ces irrégularités sont de l'ordre de la milliseconde. Par ailleurs, sur une très grande échelle de temps, la rotation de la terre sur elle-même se ralentit (de l'ordre de $20 \mu \mathrm{s} / \mathrm{an}$, soit 1 heure tous les 200 millions d'années ${ }^{2}$ ). Ces irrégularités sont évidemment contraires à la définition d'une durée de référence bien fixe dans le temps. Pour pallier à ce problème, en 1967, la durée de référence pour les mesures de temps a été redéfinie sur base d'un processus se passant à l'échelle atomique et le temps est devenu dit atomique. Très précisément, la «nouvelle » seconde a été définie comme étant précisément la durée de 9.192.631.770 périodes de la radiation correspondant à la transition entre les 2 états atomiques « $\mathrm{F}=3$ » et « $\mathrm{F}=4$ » de l'atome de césium 133. Cette «nouvelle» seconde servant dorénavant de durée de référence pour les mesures de temps s'avère être un peu plus courte que la seconde d'avant 1960 et cela oblige de temps en temps à « rajouter » de telles secondes à l'échelle de temps pour que le minuit atomique continue de coïncider au mieux avec le minuit astronomique lié à la rotation de la Terre.

Les instruments de mesure qui permettent d' « égrener les secondes atomiques » et d'effectuer de la sorte toute mesure de temps avec la nouvelle définition de la seconde en vigueur depuis 1967 sont les horloges atomiques à césium. Dans ce qui suit, nous décrivons

${ }^{1}$ Le qualificatif moyen a toute son importance car la durée exacte entre 2 passages successifs du Soleil au méridien, soit le jour solaire vrai, dépend de la période de l'année compte tenu de la trajectoire elliptique de la Terre autour du Soleil. Le jour solaire vrai varie au cours de l'année entre 23h59'39" et 24h00'30".

2 Dans 200 millions d'années, la Terre mettra de l'ordre de 25 heures de maintenant pour boucler sa rotation sur elle-même. Ce processus de ralentissement lent, mais constant, trouve son origine dans des effets de marée liée à l'interaction gravitationnelle Lune-Terre. Petit à petit, la Terre tourne de moins en moins vite sur elle-même et la Lune s'éloigne de plus en plus de la Terre (actuellement de l'ordre de $3 \mathrm{~cm} / \mathrm{an}$ ). Du fait de cet éloignement lent, mais progressif, de la Lune par rapport à la Terre, un jour les éclipses totales de Soleil ne seront plus qu'un lointain souvenir. 
les principes physiques sous-jacents au fonctionnement de telles horloges ${ }^{3}$. Le principe de base est simple : la fréquence de battement d'une horloge traditionnelle est asservie sur la fréquence caractéristique des atomes de césium à la base de la définition de la seconde. Précisons d'emblée que ces horloges, si elles sont certes affublées du qualificatif d'atomique, ne partagent en rien les connotations négatives parfois associées à ce qualificatif. Elles ne sont pas radioactives, elles ne génèrent pas de déchets radioactifs comme le font les centrales nucléaires, et elles n'explosent pas (rien en commun avec une bombe atomique). Ces horloges sont dites atomiques tout simplement parce qu'elles sont basées sur les propriétés individuelles des constituants élémentaires de la matière, à savoir les atomes. Elles sont parfaitement inoffensives et, si elles n'étaient quelque peu volumineuses, on pourrait les porter à son bracelet sans risque aucun. La figure 1 illustre l'horloge atomique à jet de césium de référence aux Etats-Unis. Celle-ci est située au NIST (National Institute of Standards and Technology, Colorado).

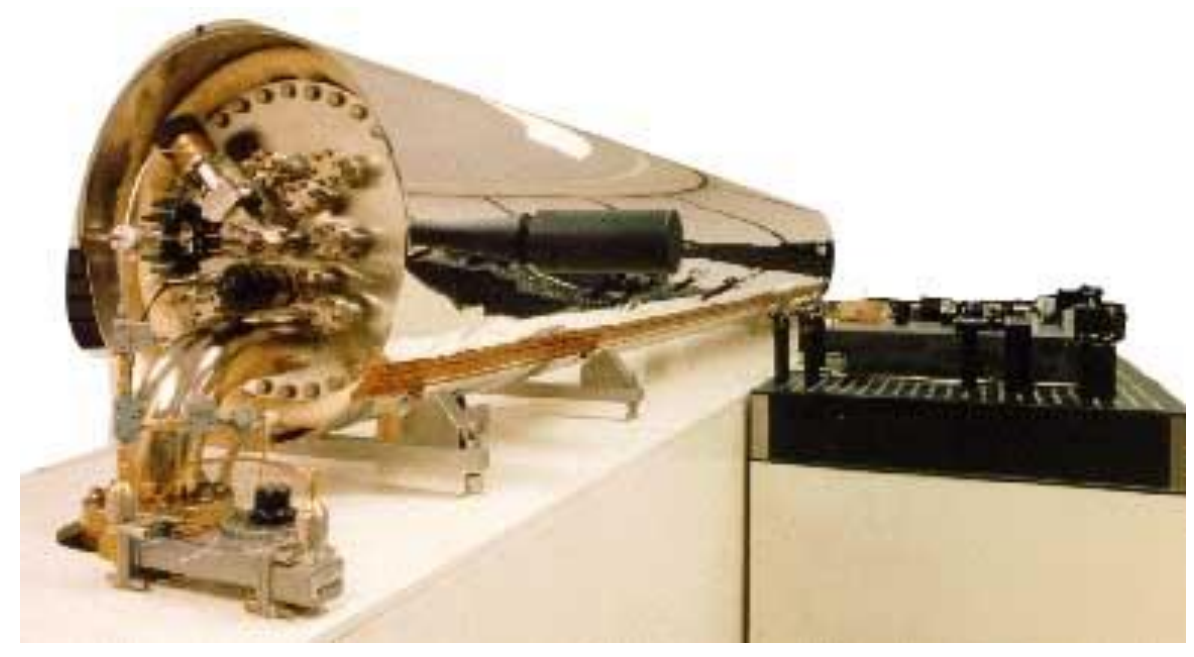

Fig. 1 : Horloge atomique de référence aux Etats-Unis (horloge NIST-7 située au NIST).

\section{Horloges à jet de césium}

Un schéma de principe d'une horloge à jet de césium est illustré sur la figure 2. Les constituants principaux sont l'oscillateur à quartz, la source de césium pour produire un jet unidirectionnel d'atomes de césium, la cavité de Ramsey et le système d'asservissement. Nous détaillons dans ce qui suit le rôle de chacun de ces constituants.

\footnotetext{
${ }^{3}$ Voir aussi notre précédent article sur le sujet dans le Bulletin de la Société Royale des Sciences de
} Liège 74, 285 (2005) duquel l'essentiel de ce qui suit est repris. 


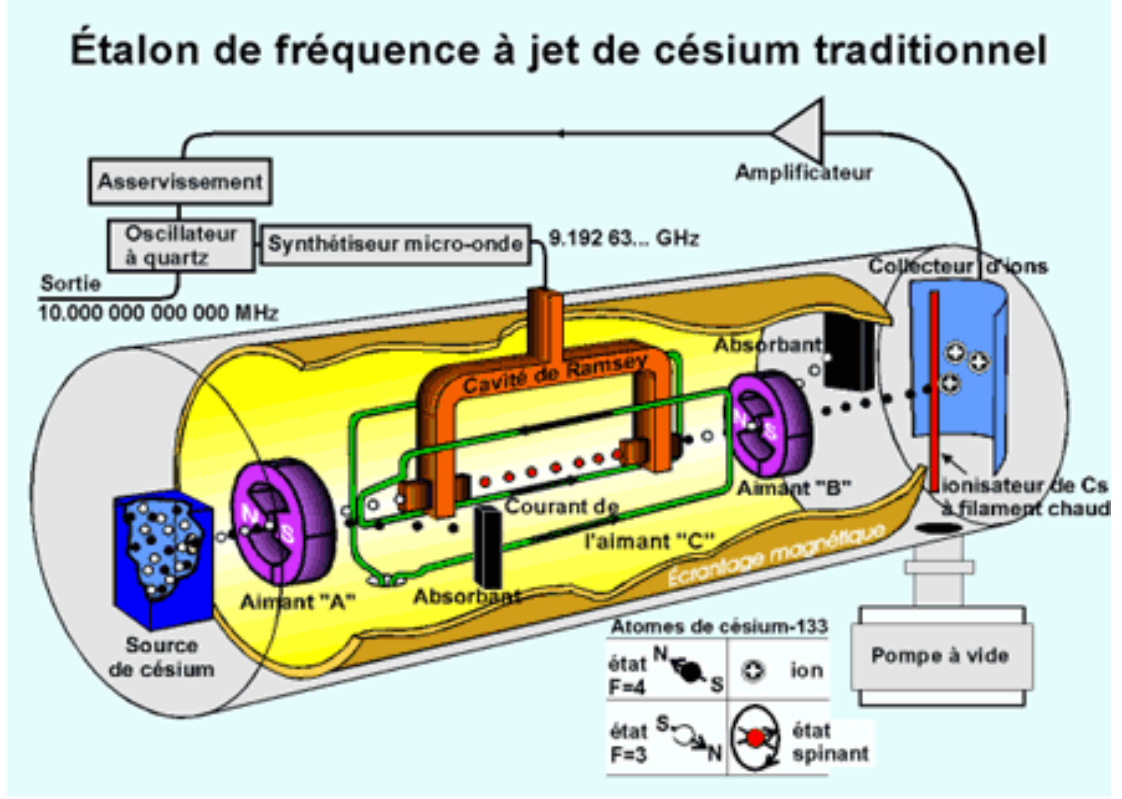

Fig. 2 : Schéma de principe d'une horloge atomique à jet de césium (figure tirée du site web de l'institut canadien des étalons nationaux de mesure, http://inms-ienm.nrccnrc.gc.ca/time_services/faq_f.html)

\section{L'oscillateur à quartz}

L'oscillateur à quartz est une horloge à quartz traditionnelle. Le principe de fonctionnement est très simple. D'une part, le quartz a la particularité de générer des charges électriques à sa surface lorsqu'on exerce sur lui des forces mécaniques (et viceversa) : c'est l'effet piézo-électrique. D'autre part, un cristal de quartz soumis à un choc engendre, comme tout cristal, des vibrations mécaniques, d'amplitude maximale selon des directions particulières (axes mécaniques) et avec une fréquence propre au quartz luimême. Il résulte de ces 2 phénomènes qu'un choc sur un cristal de quartz engendre à sa surface l'apparition de charges électriques oscillantes. Dans un circuit électronique adapté, ces oscillations peuvent être entretenues et leur fréquence peut même être légèrement modifiée. On obtient de la sorte un oscillateur électrique à une fréquence très stable pourvu que le cristal conserve ses dimensions au cours du temps. C'est le cas si le cristal est bien isolé contre les variations de température responsables des phénomènes de dilatation. Tout phénomène périodique est l'ingrédient de base d'une horloge. Il suffit de compter le nombre d'oscillations électriques du quartz et d'égrener les secondes chaque fois que ce nombre atteint une certaine valeur, caractéristique du cristal de quartz employé. Typiquement, pour des montres-bracelet à quartz, les fréquences d'oscillations sont de l'ordre de la trentaine de $\mathrm{MHz}$ (30 millions d'oscillations par seconde). 
Les premières horloges à quartz datent des années 1930. Utilisant des tubes électroniques elles avaient des dimensions comparables à celles d'un réfrigérateur. Avec l'apparition de l'électronique, la miniaturisation a rendu possible l'apparition de la première montre-bracelet à quartz en 1970. La précision obtenue est beaucoup plus grande que celle de la meilleure des montres mécaniques.

Le principal défaut d'un oscillateur à quartz est son manque de stabilité à long terme et la fréquence des oscillations se met toujours à varier. L'horloge n'égrène plus les secondes correctement et finit par retarder ou avancer selon que la fréquence diminue ou augmente. Dans une horloge à quartz traditionnelle, ces variations de fréquence ne sont pas détectées et l'horloge dérive sans que l'on puisse s'en rendre compte sans la comparer à une autre horloge qui indiquerait l'heure juste. C'est à ce niveau que se situe l'apport des horloges atomiques. Par un dispositif approprié qui fait appel à des atomes individuels, il est possible de détecter en temps réel toutes les variations de la fréquence d'oscillation du quartz et d'y pallier en agissant sur les paramètres du circuit électronique qui contrôle ces oscillations.

Dans une horloge atomique à jet de césium, l'oscillateur à quartz est couplé à un synthétiseur micro-ondes (voir figure 2). Ce dernier génère dans une cavité métallique, dite cavité de Ramsey, un rayonnement électromagnétique micro-onde, de fréquence proportionnelle à la fréquence de l'oscillateur à quartz. Toute variation, fût-elle infime, dans la fréquence d'oscillation du cristal de quartz se répercute immédiatement sur la fréquence du rayonnement micro-onde synthétisé dans la cavité de Ramsey. Les atomes, comme nous le décrivons ci-dessous, sont extrêmement sensibles au rayonnement électromagnétique qui les illumine. En faisant passer des atomes donnés dans la cavité de Ramsey, leur comportement varie selon la fréquence du rayonnement qui y règne. En détectant ce comportement, il devient possible de savoir si la fréquence d'oscillation du quartz est en train de varier ou non et de corriger ces variations dans le bon sens. Voici, grossièrement brossé, le principe de base de l'horloge atomique. Bien entendu, il existe une limite inférieure en deçà de laquelle les variations ne sont plus détectées. Cette limite fixe la précision de l'horloge atomique qui n'est évidemment pas arbitrairement élevée.

Tous les atomes d'une même espèce étant identiques les uns aux autres (rien ne ressemble plus à un atome de césium par exemple qu'un ... autre atome de césium), ceci garantit de construire des ensembles d'horloges atomiques qui oscillent toutes rigoureusement à la même fréquence, à la précision de l'horloge près. 


\section{Les atomes et le rayonnement électromagnétique. La cavité de Ramsey}

Les atomes sont des entités qui émettent ou absorbent du rayonnement électromagnétique. Cette propriété est extrêmement sélective en la fréquence du rayonnement. Une expérience toute simple permet de s'en rendre compte. Elle est illustrée sur la figure 3. La composition en fréquences (c'est-à-dire en couleurs) d'une lumière blanche est analysée au moyen d'un prisme. Lorsque cette lumière est directement envoyée sur le prisme, toutes les couleurs de l'arc-en-ciel constitutives de la lumière blanche sont obtenues au-delà du prisme. Lorsque par contre cette lumière traverse préalablement une vapeur atomique (une vapeur dont les particules constitutives sont des atomes et non des molécules - une vapeur de césium par exemple), certaines fréquences bien déterminées sont absorbées (raies sombres dans l'arc-en-ciel). Ceci démontre directement que seules certaines fréquences du rayonnement électromagnétique sont absorbées par les atomes. Ces fréquences, caractéristiques de l'espèce atomique, sont loin de se cantonner au domaine de la lumière visible. Les rayonnements $\mathrm{X}$, ultraviolets, infrarouges, micro-ondes et radio sont pareillement absorbés sélectivement par les atomes.
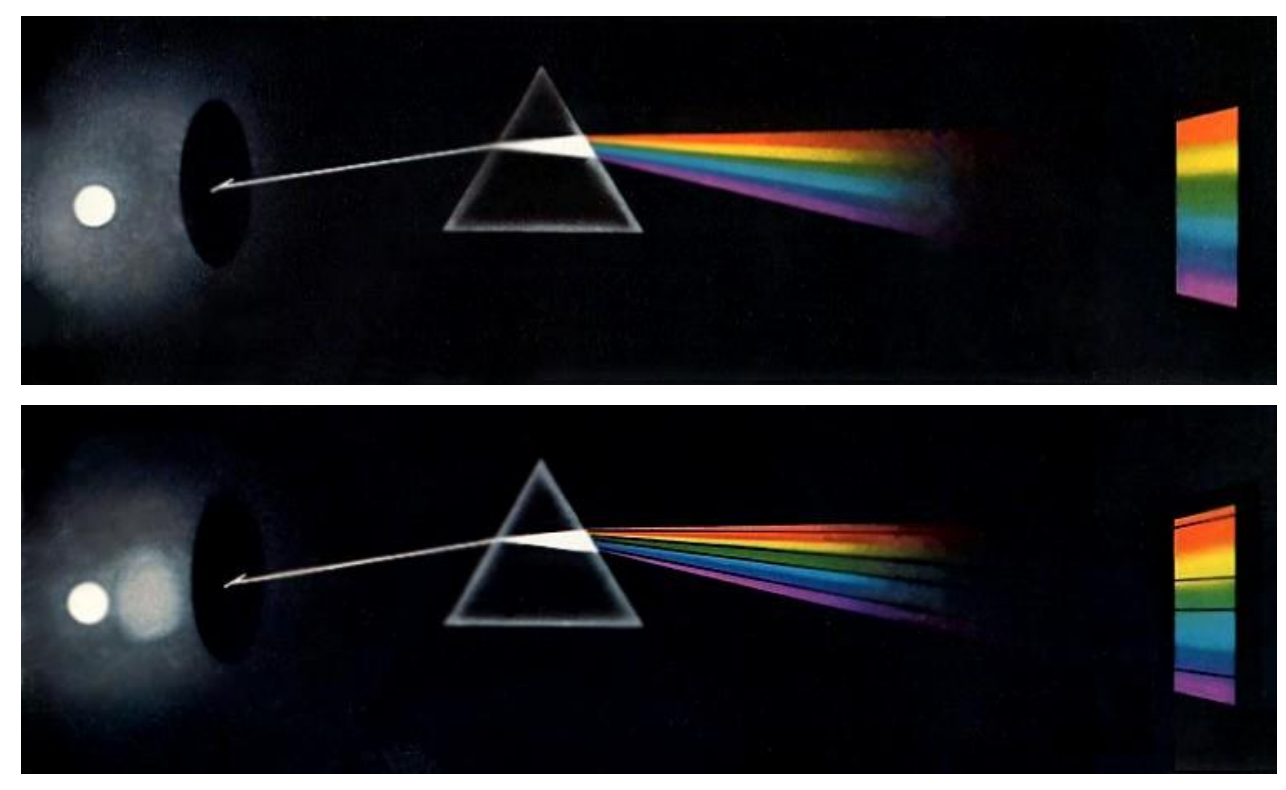

Fig. 3 : Absorption sélective de lumière blanche par une vapeur atomique.

Les atomes sont constitués d'un noyau et d'un ensemble d'électrons en mouvement autour du noyau. Ces mouvements sont contenus dans des dimensions de l'ordre du dixième de nanomètre ( 1 nanomètre $=1$ milliardième de mètre). Les électrons sont des charges électriques négatives qui possèdent également des propriétés magnétiques. Ils se 
comportent comme de petits aimants avec un pôle Nord et un pôle Sud, ce que l'on appelle le moment magnétique de spin de l'électron. Pour des atomes à plusieurs électrons, ces petits aimants s'orientent d'une manière bien définie et peuvent conférer à l'atome électriquement neutre au global une aimantation bien déterminée (le moment magnétique de l'atome).

Lorsqu'un atome absorbe ou émet du rayonnement, l'état de mouvement des électrons est affecté et l'atome est dit se trouver dans un autre état qu'initialement. Selon la fréquence du rayonnement avec lequel l'atome interagit, on porte l'atome dans tel ou tel état. L'interaction d'un atome avec du rayonnement est ainsi un moyen efficace pour changer sélectivement l'état de l'atome. On dit que l'atome effectue une transition entre 2 de ses états. Cette propriété est optimale pour certaines fréquences $v_{i}$ bien déterminées. Les physiciens, et plus précisément les spectroscopistes, donnent un nom à chacun des états possibles des atomes. Dans une vapeur atomique à une température donnée, tous les atomes ne se trouvent pas dans le même état. Dans le cas particulier des atomes de césium, ils sont soit dans l'état dénommé « F=3 », soit dans l'état « F=4 ${ }^{4}$. On fait passer ces atomes d'un état à l'autre en les éclairant avec du rayonnement électromagnétique à la fréquence de 9.192.631.770 $\mathrm{Hz}^{5}$.

La théorie quantique permet de prédire en tout instant la probabilité que l'atome se trouve dans tel ou tel état. Si un atome est initialement dans un état 1 et qu'il interagit avec du rayonnement monochromatique à la fréquence $v_{0}$ adéquate pour le porter dans un état 2 , la probabilité de trouver l'atome dans l'état 2 au cours du temps est illustrée sur la figure 4(a). Cette probabilité oscille entre 0 et 1 suivant une période $\tau$. Lorsqu'elle est égale à 1 (en $t=\tau / 2,3 \tau / 2, \ldots$ ), l'atome se trouve avec certitude dans l'état 2. Ces oscillations portent le nom d'oscillations de Rabi, du nom du physicien qui décrivit ce phénomène et qui en obtint le prix Nobel en 1944.

${ }^{4} \mathrm{Il}$ s'agit, pour être précis, des 2 états hyperfins du niveau fondamental $6 s^{2} S_{1 / 2}$ de l'atome de césium.

${ }^{5}$ Il s'agit d'une valeur exacte (sans aucune incertitude) compte tenu précisément de la définition actuelle de la seconde (l'inverse du hertz). 

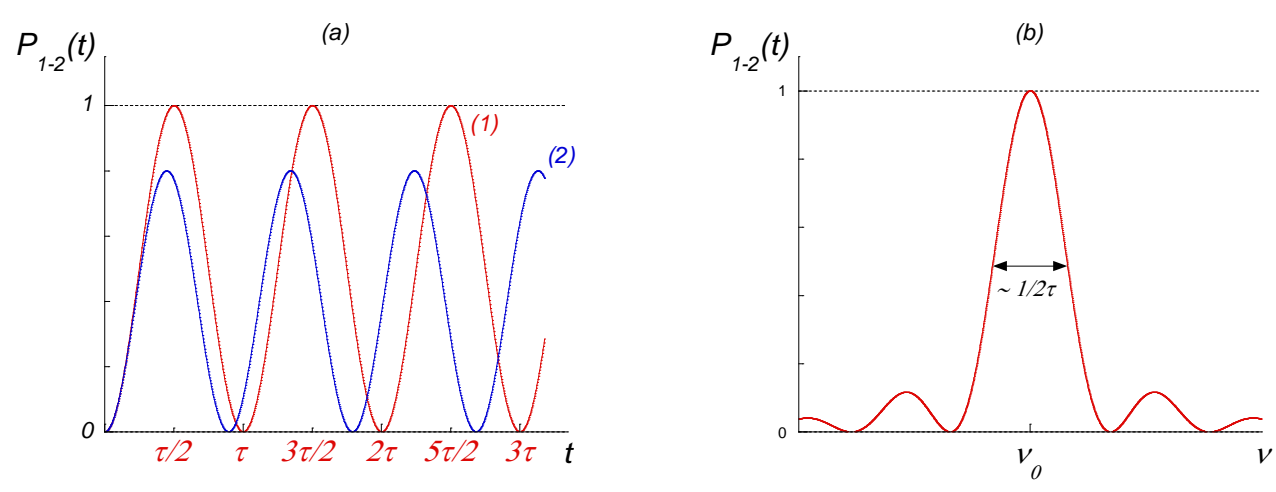

Fig. 4 : (a) Probabilité de trouver l'atome dans un état 2 en fonction du temps lorsque, initialement dans un état 1, il interagit avec du rayonnement électromagnétique (1) à la fréquence adéquate $v_{0},(2)$ à une fréquence $v \neq v_{0}$. (b) Cette même probabilité en fonction de la fréquence du rayonnement lorsque l'atome interagit pendant un temps $t / 2$.

Si la fréquence du rayonnement qui « éclaire » l'atome est légèrement modifiée par rapport à la fréquence idéale $v_{0}$, l'atome peut encore évoluer de l'état 1 vers l'état 2, mais avec une efficacité moindre (voir figure 4(a)). Pour des atomes interagissant pendant une durée $\tau / 2$ avec le rayonnement, la figure 4(b) illustre la probabilité de trouver ces atomes dans l'état 2 en fonction de la fréquence du rayonnement au voisinage de la fréquence idéale $v_{0}$. Cette probabilité décroît rapidement si la fréquence du rayonnement s'écarte de $v_{0}$ (la largeur à mi-hauteur de la courbe est de l'ordre de $1 / 2 \tau$ ). Ceci révèle que le comportement des atomes est extrêmement sensible à la fréquence du rayonnement qui les éclaire. Cette propriété rend les atomes des entités capables en quelque sorte de «mesurer » la fréquence du rayonnement. En effet, en faisant passer $N$ atomes, initialement dans un état 1 , dans une région de l'espace où règne un champ électromagnétique de fréquence proche de $v_{0}$, le nombre d'atomes que l'on trouvera dans l'état 2 au sortir de cette région est donné par $N p_{1->2}(v)$. Si $N$ est connu, on en déduit immédiatement $p_{1>2}(v)$ et par voie de conséquence la fréquence $v$ elle-même.

C'est ce procédé qui est utilisé dans une horloge atomique à jet de césium. Des atomes de césium dans l'état « F=3 » sont envoyés dans une région de l'espace (cavité de Ramsey) où règne un champ électromagnétique dont la fréquence est déterminée par la fréquence d'oscillation de l'horloge à quartz en amont. Au sortir de cette région, on mesure le nombre d'atomes ayant effectué une transition vers l'état «F=4 » et on en déduit si la fréquence dans la cavité est optimale ou non. Dans le cas contraire, un système d'asservissement agit sur la fréquence du quartz de manière à corriger les éventuels écarts en fréquence. 
Plus le temps $\tau$ d'interaction des atomes dans la cavité est élevé, plus la courbe illustrée sur la figure 4(b) est un pic étroit (puisque la largeur du pic est en $1 / 2 \tau$ ), plus fines en sont les variations de fréquences détectées et d'autant plus précise en est l'horloge. Idéalement, il est donc opportun de travailler avec des cavités d'autant plus grandes. Cela présente toutefois des inconvénients technologiques. N. Ramsey a en réalité montré qu'il était équivalent de considérer un système de 2 cavités séparées par un intervalle libre (comme le système illustré sur la figure 2). Dans ce cas, il s'avère que la largeur à mihauteur de la courbe de la figure 4(b) devient inversement proportionnelle au temps de parcours $T$ des atomes entre les 2 cavités et c'est ce seul paramètre qui détermine la précision de l'horloge. Pour des horloges atomiques «standards », ce temps de parcours est de l'ordre de la milliseconde et la courbe de probabilité 4(b) a une largeur à mi-hauteur de l'ordre de la centaine de Hertz. Ceci permet de se rendre compte avec quelle précision la fréquence de $9.192 .637 .770 \mathrm{~Hz}$ peut être déterminée. Dès que cette fréquence varie de quelques hertz dans la cavité de Ramsey, le nombre d'atomes dans l'état « F=4 » chute et l'on sait que la fréquence d'oscillation du quartz vient de varier suivant un même rapport (quelques hertz sur 9,192 milliards de hertz, soit dans un rapport de l'ordre de $10^{-9}$ ). Le système d'asservissement peut entrer en action pour rattraper cette dérive. Actuellement les meilleures horloges atomiques à jet de césium déterminent la seconde à $10^{-14}$ près.

\section{La source de césium}

Pour produire un jet d'atomes de césium, on chauffe tout simplement dans une enceinte fermée (le four sur la figure 2) un échantillon de césium pur. Sous l'effet de la chaleur, le césium se vaporise. Par un canal de sortie percé dans la paroi du four, les atomes présents dans la vapeur s'échappent dans la direction définie par l'axe du canal et définissent de la sorte un jet d'atomes directionnel. Les atomes qui s'échappent ont une vitesse typique de l'ordre de $250 \mathrm{~m} / \mathrm{s}$.

Le césium est un métal alcalin, de numéro atomique 55 (ce numéro correspond au nombre d'électrons autour du noyau) et découvert en 1861 par Bunsen et Kirchhof. C'est un métal très mou de couleur or argenté (voir figure 5). Sa température de fusion est de $28,4{ }^{\circ} \mathrm{C}$ et d'ébullition de $669^{\circ} \mathrm{C}$. Le césium s'oxyde rapidement dans l'air et peut causer des brûlures très graves quand il entre en contact avec la peau. Un gramme de césium suffit pour faire fonctionner une horloge atomique pendant une année entière. 


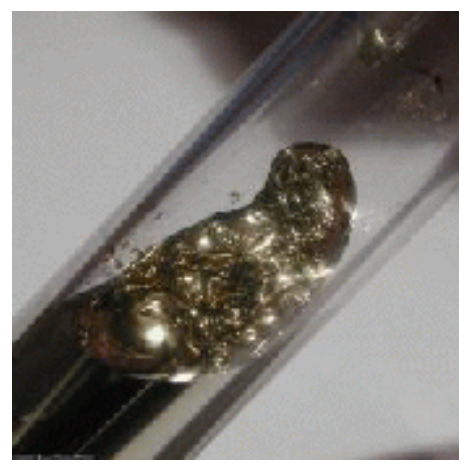

Fig. 5 : Echantillon de césium pur.

Dans le four, on trouve 50\% d'atomes de césium dans l'état « F=3 » et 50\% dans l'état « $\mathrm{F}=4$ ». Les atomes dans ces 2 états ont des propriétés magnétiques différentes. Dans un champ magnétique, ils se comportent comme de petits aimants qui s'orientent différemment. C'est pourquoi, à la sortie du four, les atomes de césium sont envoyés dans 1'entrefer d'un aimant où règne un champ magnétique ${ }^{6}$. Dans ce champ magnétique, les atomes suivent des trajectoires différentes selon leur état. Seuls les atomes dans l'état « $\mathrm{F}=3$ » sont envoyés dans la cavité de Ramsey où règne le rayonnement électromagnétique. A la sortie de la cavité, les atomes peuvent avoir effectué la transition vers l'état « F=4 » suivant la probabilité donnée par la figure 4(b). On distingue les atomes ayant effectué cette transition des autres en les faisant passer au travers d'un second aimant. Seuls les atomes dans l'état « $\mathrm{F}=4$ » sont envoyés vers un détecteur à atomes pour y être comptés (voir figure 2) afin d'en déduire la fréquence du rayonnement électromagnétique régnant dans la cavité de Ramsey.

\section{Horloges à atomes froids}

La précision d'une horloge atomique telle que celle illustrée sur la figure 2 est déterminée par un seul paramètre : le temps de parcours $T$ des atomes entre les 2 branches de la cavité de Ramsey. Pour des atomes de césium issus d'un four, les vitesses typiques des atomes sont de l'ordre de la centaine de mètres par seconde et les temps $T$ pour les atomes sont par conséquent de l'ordre de la milliseconde (les 2 branches de la cavité de Ramsey sont typiquement séparées d'une distance de l'ordre de quelques dizaines de centimètres, jamais beaucoup plus). Il est extrêmement difficile d'augmenter significativement ce temps $T$ (et par conséquent la précision de l'horloge) sur base de ce schéma de principe.

\footnotetext{
${ }^{6}$ Pour être précis, il doit s'agir d'un gradient de champ magnétique.
} 

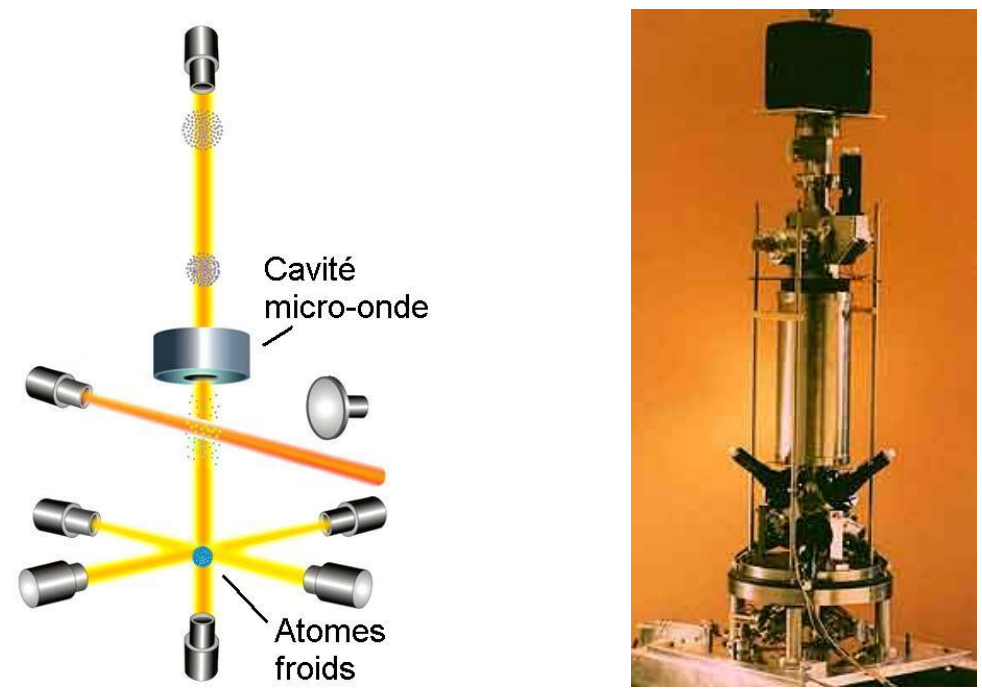

Fig. 6 : Schéma de principe d'une horloge atomique à « atomes froids ». Ces derniers sont produits à l'intersection des 6 faisceaux lasers (partie inférieure de la figure). Photo de droite : une des horloges atomiques à atomes froids du laboratoire Kastler Brossel à Paris, développée par C. Salomon et al.

L'avènement de la technologie des «atomes froids » a fortement changé la donne ces dernières années. Les atomes froids sont des atomes d'une vapeur que l'on fait interagir avec divers faisceaux laser. Sous une géométrie particulière (voir figure 6) et pour certaines longueurs d'onde (c'est-à-dire couleurs) bien déterminées du laser, cette interaction donne lieu à un refroidissement de la vapeur à des températures extrêmement basses (de l'ordre de quelques millionièmes de degrés seulement au-dessus du zéro absolu, soit $-273,15{ }^{\circ} \mathrm{C}$ ). A de telles températures, les atomes sont quasiment immobilisés et restent suspendus dans le vide par la lumière. Il devient possible de les sonder, les contrôler et les manipuler (même individuellement) avec une extrême précision. Ces possibilités nouvelles ont ouvert la voie à de nombreuses perspectives de recherche tant fondamentales qu'appliquées. Ces domaines de recherche ont été couronnés par plusieurs prix Nobel de physique (C. CohenTannoudji, S. Chu, W. D. Phillips en 1997, et E. A. Cornell, C. A. Wieman, W. Ketterle en 2001).

Dans une horloge atomique à «atomes froids", les atomes immobilisés à l'intersection des faisceaux laser sont « lancés » vers le haut avec une certaine vitesse (par modification de la fréquence des lasers verticaux). En coupant ensuite tous les faisceaux laser, les atomes lancés verticalement continuent leur mouvement d'ascension, atteignent une hauteur maximale, puis retombent vers le bas, ... exactement de la même façon qu'une pierre lancée verticalement finit toujours par retomber vers son point de départ (la gravité 
agit sur tous les corps à l'identique indépendamment de leur masse ${ }^{7}$ ). On réalise de la sorte ce que l'on appelle des fontaines atomiques. Dans leur mouvement vertical, les atomes traversent à 2 reprises une cavité micro-onde (une fois à la montée et une seconde fois lors de la redescente). On reproduit de la sorte le même schéma de principe de l'horloge à jet de césium où l'on fait passer des atomes de césium successivement au travers de 2 cavités micro-onde. Cependant, dans le cas présent, l'intervalle de temps $T$ entre les 2 passages des atomes au travers de la cavité micro-onde peut être rendu significativement plus long, typiquement de l'ordre de la seconde ${ }^{8}$ (c'est mille fois plus que dans le cas des horloges à jet de césium). Il en résulte une horloge atomique avec une précision nettement accrue. Les horloges à atomes froids utilisent couramment des atomes de rubidium en lieu et place des atomes de césium.

Il existe actuellement un projet d'envoyer une horloge atomique à atomes froids

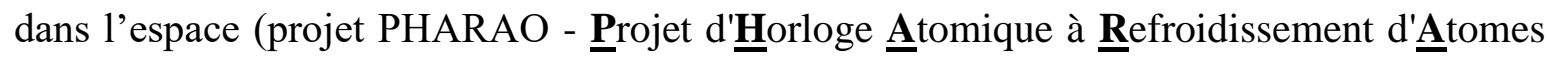
en Orbite). L'intérêt réside en ce que l'absence de gravité peut encore contribuer à augmenter le temps $T$ et ainsi la précision de l'horloge. L'objectif est d'atteindre une précision de $10^{-16}$, contre $10^{-15}$ actuellement pour les meilleures horloges à atomes froids sur Terre.

Les prototypes des horloges PHARAO sont testés dans des expériences de microgravité avec l'avion zéro-G d'Airbus (figure 7). Cet avion reproduit dans son intérieur un environnement exempt de gravité pendant quelques secondes. A cette fin, l'avion entame un mouvement ascensionnel fort incliné en haute altitude puis coupe tous ses moteurs. L'avion poursuit alors un mouvement parabolique et « tombe » littéralement en chute libre. Pendant ces instants, tout se passe à l'intérieur comme si la gravité n'existait pas. Après une vingtaine de secondes, et pour d'évidentes raisons, les moteurs sont remis en marche et de nouvelles paraboles peuvent être entreprises. Une campagne de vols paraboliques comporte une trentaine de paraboles. Il est évidemment préférable de ne pas tomber malade dès les premières minutes de vol (cela arrive pourtant fréquemment d'après les récits des expérimentateurs prenant part à ces campagnes de mesures).

\footnotetext{
${ }^{7}$ Dans le vide, une pierre et une plume tombent côte à côte. Avec un seul atome en lieu et place de la plume, on obtiendrait exactement le même comportement.

${ }^{8} \mathrm{C}$ 'est le temps caractéristique qu'il faut à un atome pour monter d'une soixantaine de centimètres et redescendre de cette même hauteur.
} 


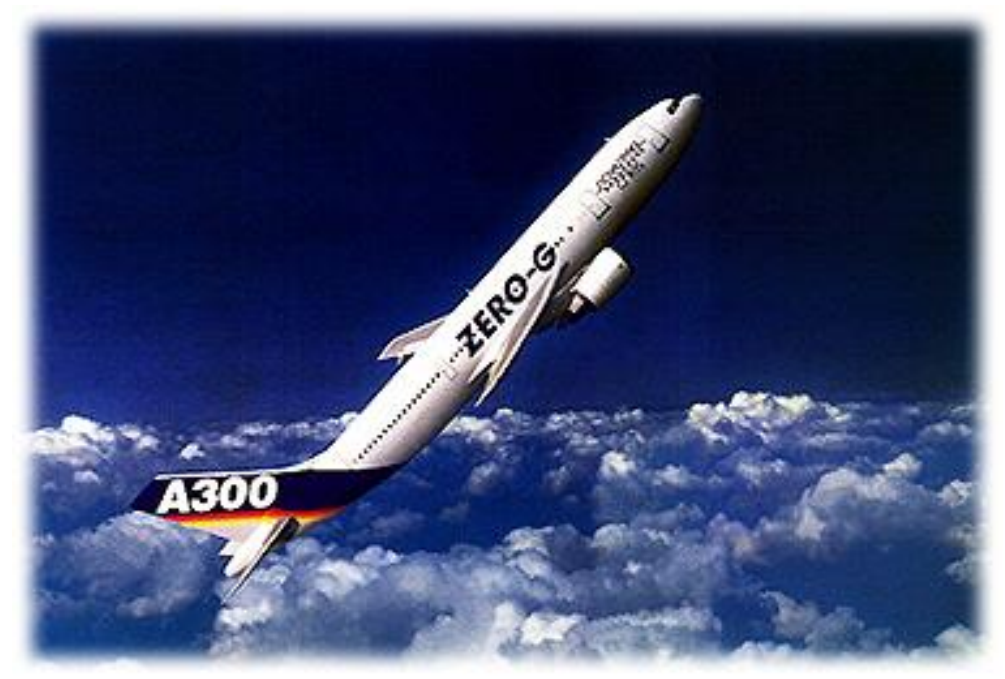

Fig. 7 : Avion Zero-G d'Airbus pour les campagnes de vols paraboliques et les expériences en micro-gravité.

\section{Conclusions}

Les horloges atomiques sont des appareillages scientifiques de haute technologie en perpétuelle évolution. Les dernières générations de ces appareils font appel aux développements récents de la recherche en physique atomique et l'on peut s'attendre à voir dans les prochaines années la définition de la seconde révisée, tant la précision sur la détermination du temps s'est accrue depuis 1967, date de l'actuelle définition. Les physiciens atomiques sont continuellement à la recherche de systèmes sensibles à des variations de plus en plus fines de la fréquence du rayonnement électromagnétique qui les illumine.

Les horloges atomiques ont contribué à mettre en évidence des phénomènes qui continuent à nous surprendre aujourd'hui, tels par exemple la relativité du temps. Le temps d'un système que l'on voit bouger s'écoule plus lentement. Une horloge mise en mouvement et ramenée à son point de départ affiche un retard comparativement à une horloge qui serait restée au repos à ce même point de départ. Nul espoir de justifier un retard à une réunion en invoquant ce phénomène, ces effets sont extrêmement ténus pour des objets se déplaçant aux vitesses usuelles de notre environnement quotidien. Une horloge embarquée dans un avion se déplaçant à $1.000 \mathrm{~km} / \mathrm{h}$ retarde de quelque 0,4 millième de milliardième de seconde par seconde de temps écoulée au sol. Si le vol dure 1 heure à cette vitesse, l'horloge voyageuse affichera un retard de 1,5 milliardième de seconde par rapport à une horloge restant au sol. Seules les expériences réalisées au moyen d'horloges atomiques ont été capables de mettre en évidence et de confirmer ces effets de 
relativité restreinte (théorie sur la nature de l'espace et du temps établie par Einstein en 1905). Pour produire des retards plus importants, il faudrait faire se mouvoir des horloges avec des vitesses beaucoup plus grandes. Les effets les plus significatifs ont lieu lorsque la vitesse de déplacement approche celle de la lumière $(300.000 \mathrm{~km} / \mathrm{s})$.

De manière analogue, le temps ne s'écoule pas de la même façon selon l'altitude du point où l'on se trouve. Il s'agit là d'un effet de relativité générale (théorie de la relativité qui prend en considération le phénomène de gravitation - l'attraction universelle de tous les corps entre eux). Il faut des variations d'altitude de l'ordre de 10.000 mètres pour obtenir des effets du même ordre de grandeur que ceux obtenus en embarquant une horloge dans un avion à $1.000 \mathrm{~km} / \mathrm{h}$. Encore une fois, ces effets ont pu être mesurés et confirmés au moyen d'horloges atomiques.

Le système GPS utilise des signaux provenant de satellites situés à $20.000 \mathrm{~km}$ d'altitude. Les horloges atomiques embarquées dans ces satellites mesurent un temps qui s'écoule donc différemment que sur Terre. Non seulement elles sont parfaitement à même de détecter cette différence, mais en outre elles doivent prendre en compte ces effets de relativité générale pour éviter de rendre immédiatement caduque le fonctionnement du GPS. Qui imagine un seul instant que, se promenant en forêt au moyen de son récepteur GPS, les équations de la relativité générale sont encodées dans ce récepteur et que ce sont des horloges atomiques situées à $20.000 \mathrm{~km}$ d'altitude qui lui permettent de suivre son chemin sans se tromper? Les recherches à caractère fondamental ont parfois des retombées technologiques insoupçonnées bien des années plus tard. La relativité générale fut développée en 1916 par Einstein et les effets prédits sont à ce point ténus sur Terre qu'à cette époque on ne s'imaginât jamais qu'un jour et une septantaine d'années plus tard des applications bien concrètes en découleraient. La détermination précise du temps est et continuera à l'avenir d'être un sujet de prime importance dont les répercussions sur la recherche fondamentale et partant la recherche appliquée ne se démentiront certainement pas. 